\title{
A case of ovarian torsion of mixed epithelial tumor misdiagnosed as a malignancy in postmenopausal woman
}

\author{
Jeon Hyeji, Ryu Aeli \\ Obstetrics and Gynecology, Soonchunhyang university Cheonan hospital, Cheonan, \\ Dongnam-Gu, South Korea
}

\section{Problem statement:}

Adnexal torsion is often diagnosed in reproductive age but rare in postmenopausal women at approximately $2.7 \%$. So most cases of adnexal torsion with postmenopausal women are diagnose as a malignancy preoperatively. The recent case is presented that ovarian torsion with mixed epithelial tumor misdiagnosed as a malignancy in postmenopausal woman.

\section{Methods:}

We present a case of ovarian torsion of mixed epithelial tumor misdiagnosed as a malignancy in postmenopausal woman

\section{Results:}

A patient aged 65 years presented with lower abdominal pain which started 3 days ago. Ultrasound showed a multiseptated cystic mass with a solid portion in the left ovary. So malignancy was suspected but serum CA-125 revealed normal levels. The patient underwent exploratory laparotomy. A white-gray cystic tumor was detected in the left adnexa, having a torsion of $720^{\circ}$ in a counterclockwise direction. First, the left salpingooophorectomy was performed and the result of a frozen section biopsy confirmed a borderline Brenner tumor associated with a mucinous tumor. Subsequently, total hysterectomy and right salpingo-oophorectomy were performed.

The specimen revealed that the cyst wall was lined by a single layer of benign mucinous cells, which was consistent with mucinous cystadenoma. The solid area displayed round to oval tumor cell nests within fibrous stroma, obvious nucleoli and

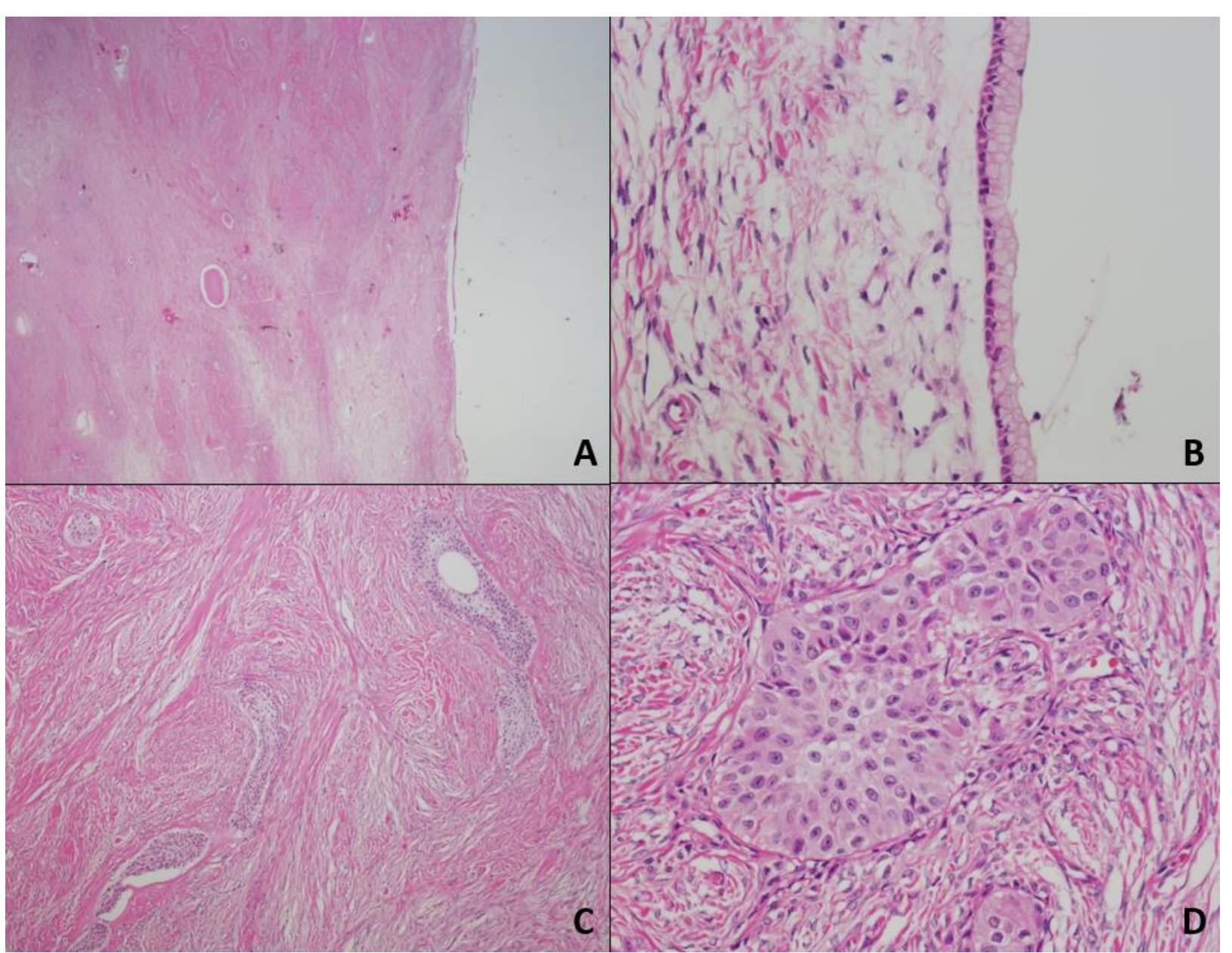

longitudinal grooving with no cytologic atypia, of which features were benign Brenner tumor (Figure).

\section{Conclusion:}

Adnexal torsion in postmenopausal women is difficult to diagnose preoperatively because the clinical symptom of it is constant and dull in postmenopausal women. Furthermore, the risk of malignancy increases with age in epithelial ovarian tumors. The patient of this case shows $s$ a large, multicystic ovarian mass containing a solid portion in radiologic assessment. Because mucinous tumors are usually large in size, manifest as multicystic lesions and Brenner tumors appear as a solid mass, discrimination between benign and malignancy is difficult with sonographic findings alone.

Due to these reasons, surgery is performed based on condition severity in preparation for malignant tumor treatment instead of torsion. But it should be taken into consideration that the possibility of malignancy is low in postmenopausal women with normal CA125 levels and although very rare, ovarian torsion can occur in postmenopausal women, This consideration makes us carry out appropriate surgery based on the result of frozen section biopsy to exclude the possibility of malignancy.

It is important that although malignant ovarian tumor is suspected preoperatively in postmenopausal women, surgeons can avoid unnecessary surgical procedures by considering the possibility of benign when there is intraoperative finding of ovarian torsion.

Figure. (A) The tumor consisted of solid (left side) and cystic (right side) areas. (H\&E, x40) (B) Cystic spaces were covered with single layered mucinous cells. (H\&E, x400) (C) Solid areas showed dense fibrous stroma and round to oval shaped epithelial cell nests. (H\&E, x100) (D) Tumor cell nests in solid areas showed no abundant eosinophilic cytoplasms with a single prominent nucleoli and frequent nuclear groove. (H\&E, x400) 